\title{
E-learning en tiempos de COVID. La experiencia del CLUSTER de Enseñanza -Aprendizaje AULACIETE 2020-2021
}

\section{E-learning in times of COVID. The experience of the AULACIETE 2020- 2021 Teaching-Learning CLUSTER}

\author{
Raymond Marquina \\ raymond@aulaciete.net \\ https://orcid.org/0000-0003-1357-4436 \\ Universidad de los Andes, Mérida, Venezuela \\ Ángel Alvarado \\ alvaradoangel07@aulaciete.net \\ https://orcid.org/0000-0002-1169-3688 \\ AULACIETE \\ Elisabeth Benítez \\ elisabeth.benitez@aulaciete.net \\ https://orcid.org/0000-0002-1643-823X
}

AULACIETE

Recibido: 06/04/21

Aceptado: 28/05/21

\section{Resumen}

La pandemia por COVID-19 hizo que el acceso a la educación se viera afectada. Del análisis a las medidas gubernamentales, se constató la ausencia de capacitación docente, esto motivó la iniciativa de un grupo de Profesionales para generar un programa en línea. El objetivo de este artículo es: Señalar los elementos que conformaron la iniciativa; Identificar los resultados obtenidos; Resaltar los factores de éxito y emitir algunas recomendaciones. La investigación es cualitativa, de tipo descriptivo. Se destaca la necesidad de dotación de equipos, uso de aplicaciones especializadas, Internet y espacios para el desarrollo de competencias en la autogestión del aprendizaje.

Palabras clave: E-learning, COVID 19, Cluster de Enseñanza - Aprendizaje, AULACIETE. Formación en línea.

\section{Abstract}

The COVID-19 pandemic affected access to education. From the analysis to the governmental measures, the absence of teacher training was found, this motivated the 
initiative of a group of Professionals to create an online program. The objective of this article is: To point out the elements that made up the initiative; Identify the results obtained; Highlight the success factors and make some recommendations. The research is qualitative, descriptive. The need to provide equipment, use of specialized applications, the Internet and spaces for the development of skills in self-management of learning is highlighted.

Keywords: E-learning, COVID 19, Teaching - Learning Cluster, AULACIETE. Online training.

\section{Introducción}

Con el reconocimiento que da la Organización Mundial de la Salud (OMS) a la pandemia por COVID19 el 11 de marzo de 2020 se activaron una serie de acciones gubernamentales a nivel mundial entre las cuales destacó el denominado "aislamiento social" que trajo consigo el cierre de todos los establecimientos educativos en los distintos niveles, medida que se acogió y adoptó de forma prácticamente absoluta en los países miembros de la región latinoamericana y del caribe.

Ante la recomendación de la Organización de las Naciones Unidas para la Educación, la Ciencia y la Cultura (IESALC-UNESCO, 2020) acerca del uso de sistemas de educación a distancia y el aprovechamiento ofertado por las tecnologías digitales, se incrementaron las brechas de acceso educativo y tecnológico, y por ende, la crisis sanitaria agravó circunstancias sociales críticas que existían previamente a la pandemia.

Entre ellas destaca el hecho de que la educación en la región se ha caracterizado por la inequidad en el acceso de los estudiantes, lo que vino a reforzar necesidades detectadas con antelación como las que exponen Reimers, F. M., \& Schleicher, A. (2020) quienes refieren la necesidad de acceso en los contextos más vulnerables, así como recalcan la importancia de la calidad de las herramientas didácticas, tecnológicas y materiales que se pudieran brindar a las familias, docentes y directivos para garantizar el aprendizaje de los estudiantes desde sus casas.

Para mitigar la situación de emergencia educativa, los gobiernos del mundo ofrecieron una serie de lineamientos, políticas y medidas tendentes a mitigar las circunstancias pre - existentes agravadas por la situación, esto permitió conocer algunas deficiencias antes detectadas, pero que se vieron exacerbadas gracias a la necesidad de migrar enteramente a una modalidad de educación en línea ( $e$-learning), como lo fue la insuficiente capacitación en estrategias didácticas y en el uso de herramientas tecnológicas en la mayoría de los docentes de los distintos niveles educativos de la región.

Por ello, paralelo a las iniciativas gubernamentales, un grupo de docentes venezolanos, asociados a una organización no gubernamental, tomaron la determinación de desarrollar un programa de formación y actualización docente en herramientas didácticas

\footnotetext{
56 E-learning en tiempos de COVID. La experiencia del CLUSTER de Enseñanza -Aprendizaje AULACIETE 2020 - 2021.
} 
y tecnológicas, denominado "Clúster de Enseñanza y Aprendizaje AULACIETE (CLEAP7)", que promoviese el adecuado uso de las tecnologías de información y comunicación, desarrollado bajo la modalidad de formación interactiva a distancia con acceso abierto, masivo y gratuito, con la particularidad de contar con un diseño modular del contenido que les permitiese a los participantes la autogestión de su formación.

Este artículo tiene por objeto describir el proceso de diseño, desarrollo y gestión del CLEAP-7 en el periodo comprendido entre septiembre 2020 y febrero 2021, además de identificar los principales resultados obtenidos durante el desarrollo del proceso, mediante la realización de un análisis de Fortalezas, Debilidades, Oportunidades y Desafíos, resaltando los factores críticos de éxito a partir de los resultados obtenidos con la participación de 530 docentes registrados en el CLEAP-7, y finalmente, pretende presentar sugerencias a proyectos de formación de similar naturaleza.

\section{Planteamiento del problema}

Según datos de la Organización de las Naciones Unidas para la Educación, la Ciencia y la Cultura (UNESCO) a mediados de mayo de 2020 más de 1.200 millones de estudiantes de todos los niveles de enseñanza en todo el mundo, habían dejado de tener clases presenciales, de ellos, más de 160 millones eran estudiantes de América Latina y el Caribe (CEPAL,2020) En consecuencia, los efectos nocivos de la pandemia por COVID a nivel social han sido profundos en el ámbito educativo y social.

Adicionalmente, la UNESCO ha determinado que se han profundizado las brechas en los resultados educativos debido a que: "existe una desigual distribución de los docentes, en general, y de los docentes mejor calificados, en particular, en desmedro de países y regiones con menores ingresos y de zonas rurales, las que suelen concentrar además a población indígena y migrante" (UNESCO, 2016a; Messina y García, 2020 cit por CEPAL)

Como consecuencia, los gobiernos de la región Latino Americana se vieron ante la necesidad de tomar medidas urgentes de atención, por lo que de acuerdo a sus posibilidades, cada país de la región desarrolló durante los tres últimos trimestres del año 2020 e inicios del 2021 diversas soluciones apoyadas en el uso de las tecnologías de información y comunicación (TIC) para atender las necesidades educativas que surgen de la suspensión de las clases presenciales. En algunos casos se trata de protocolos, en otros de guías de estudios descargables, plataformas digitales y el uso de medios de comunicación: radio y televisión principalmente, en los más avanzados comprendió la entrega de dispositivos móviles de apoyo.

Las iniciativas gubernamentales dan cuenta de un conjunto diverso y heterogéneo de iniciativas que la UNESCO en su "Informe de sistematización de los sistemas educativos de América Latina a la crisis de la COVID 19" (2020) intentó agrupar evaluando distintos factores en 4 tipos de iniciativas, a fin de categorizar y difundir las principales respuestas 
que han dado los distintos países de América Latina y el Caribe desde sus respectivos sectores educativos.

Como resultado del análisis anterior, se observó que la atención a la emergencia educativa a nivel gubernamental dejó un vacío importante al no considerar primariamente la capacitación, adiestramiento y formación didáctica y tecnológica a los docentes, lo cual representa uno de los principales factores de profundización de la brecha en los resultados educativos de los países de la región, dado que además: " Las escuelas que se encuentran en contextos más vulnerables tienen a los docentes con menos habilidades para integrar dispositivos digitales en la instrucción (55\%), comparado con el $68 \%$ de las escuelas con entornos más favorecidos. En general, los datos de PISA muestran evidencia sobre las necesidades de capacitación en la tecnología educativa que requieren las escuelas" (Arias \& Vitteri, 2019)

La lógica consecuencia de los factores antes mencionados fue la proliferación de "nuevos expertos" vendedores soluciones "mágicas" propiciando la generación de un proceso de "fatiga virtual" resultante de la inexperta utilización de las herramientas tecnológicas y el surgimiento de un fenómeno denominado "enseñanza remota de emergencia" (Hodges et al., 2020).

\section{Objetivos de la investigación}

A partir de lo anteriormente expuesto, la presente investigación pretende

- Señalar los elementos cardinales que conformaron la iniciativa para el proceso de desarrollo de la oferta educativa gratuita y de libre acceso CLEAP - 7 que se desarrolló entre septiembre 2020 y febrero 2021.

- Identificar los resultados obtenidos durante el desarrollo del proceso, mediante la realización de un análisis de Fortalezas, Debilidades, Oportunidades y Desafíos (FDOD) del aprendizaje en línea durante la pandemia.

- Resaltar los Factores Críticos de Éxito (FCE) para el e-learning a partir de los resultados obtenidos con la participación de 530 docentes registrados en el CLEAP 7.

- Emitir algunas sugerencias y recomendaciones para el éxito de un programa de formación e - learning.

\section{4. $\quad$ Antecedentes}

La idea del Clúster de Enseñanza y Aprendizaje nace como el resultado de las experiencias adquiridas a lo largo de más de 16 años de trabajo ininterrumpido de sus autores, en diferentes proyectos de carácter institucional y nacional en el campo de la educación a distancia y el conocimiento libre en Venezuela. 
Esta suma de experiencias, conocimientos y productos constituye la base fundamental que dio vida al proyecto del Clúster de Enseñanza y Aprendizaje de AULACIETE (CLEAP-7) un oferta de formación gratuita, libre, accesible a través de la web que surge para dar respuesta a una necesidad claramente identificada y que a tenor de lo sugerido por Reimers, F. M., \& Schleicher, A. (2020) quienes establecen como parte de su marco guía para atender la pandemia de COVID 19, que "es fundamental facilitar la colaboración y el aprendizaje profesional de los docentes, y proporcionarles acceso a recursos y plataformas en línea para la colaboración (tecnología y recursos educativos curados"

\section{Metodología}

La investigación presenta un enfoque metodológico de naturaleza cualitativa, el estudio que se presenta es del tipo descriptivo, concentrado en una muestra de 530 docentes de distintos sectores: educación básica, media, diversificada, universitaria, industrias, empresas y emprendedores pertenecientes a una población de 15 países de la región Latinoamericana y del Caribe quienes aceptaron voluntariamente participar en el CLEAP - 7, el estudio se realizó entre los meses de septiembre 2020 y febrero 2021.

Los datos se recolectaron a partir de la información reportada a través de la plataforma Moodle, de aulaciete.net, en la cual se desarrolló el CLEAP- 7 en los meses indicados, mediante el trazado de la actuación de cada uno de los participantes durante el lapso en estudio. El procedimiento de investigación consistió en el seguimiento realizado a la evolución en la participación de cada uno de los docentes involucrados.

La documentación del proceso se realizó mediante el vaciado de la información en hojas de Excel, que permitieron identificar los aspectos relevantes del estudio, a fin de responder a los objetivos previstos en esta investigación.

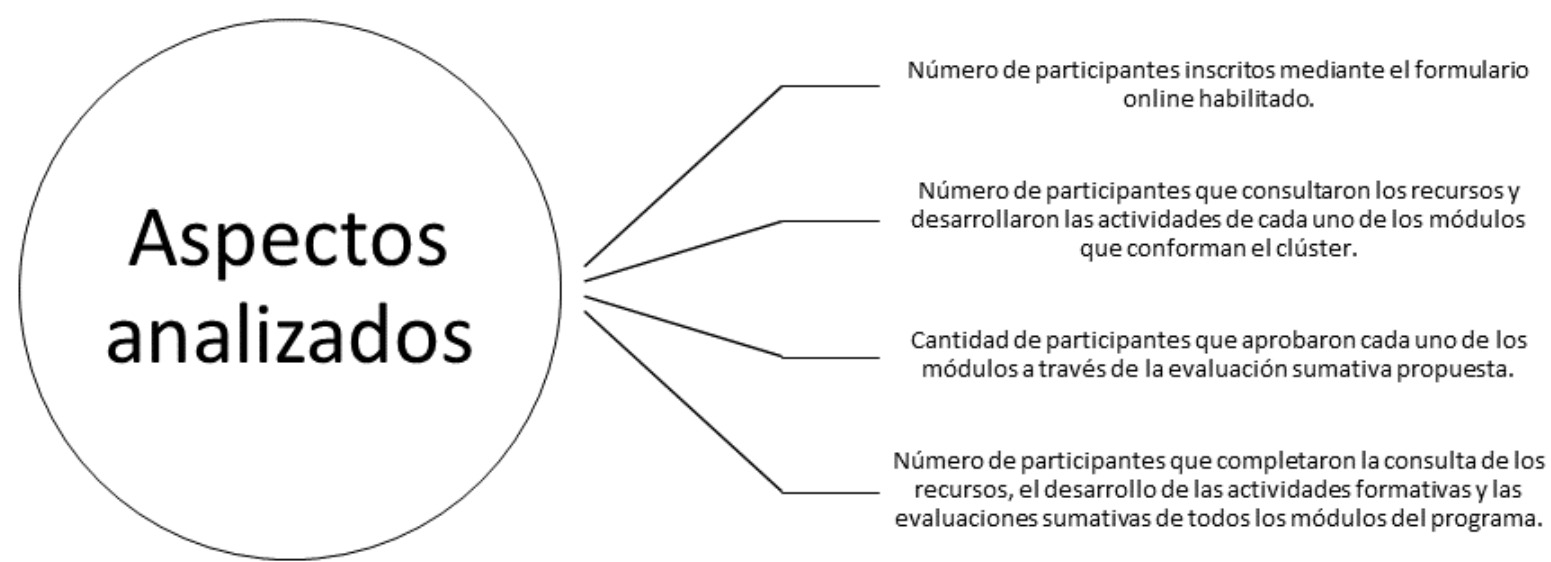




\section{Resultados}

\section{Participación}

Mediante el análisis de los reportes se ha determinado que de 530 participantes hubo un alto interés de participar en el género femenino.

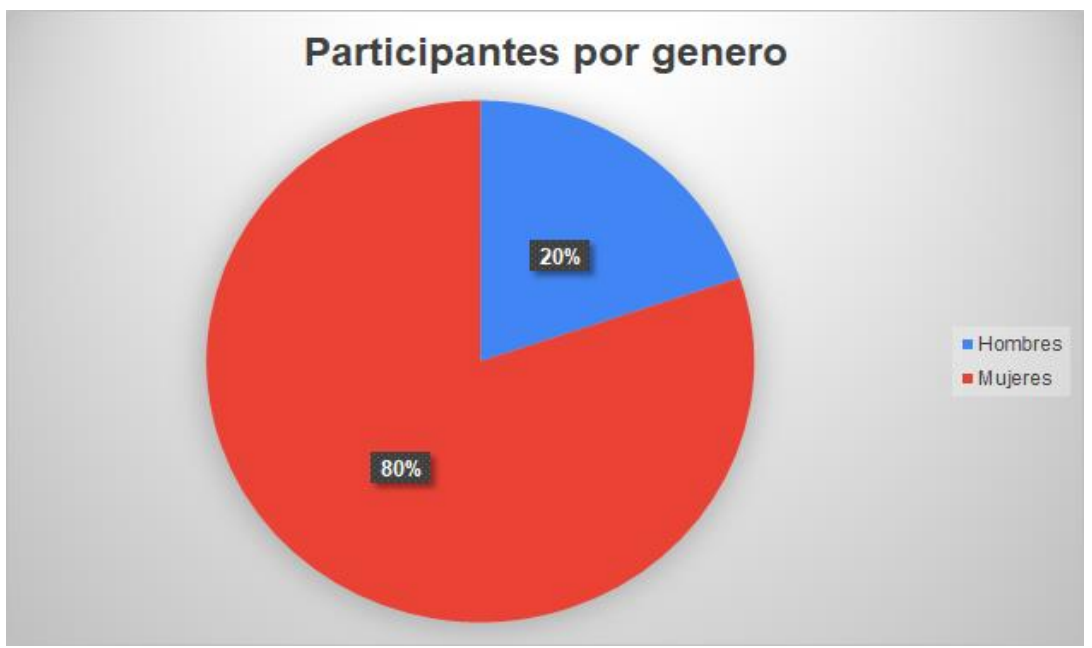

Gráfico 1. Fuente propia.

Los participantes se presentaron de 13 países de la Región de Latinoamérica y el Caribe, agregando la participación de México y España.

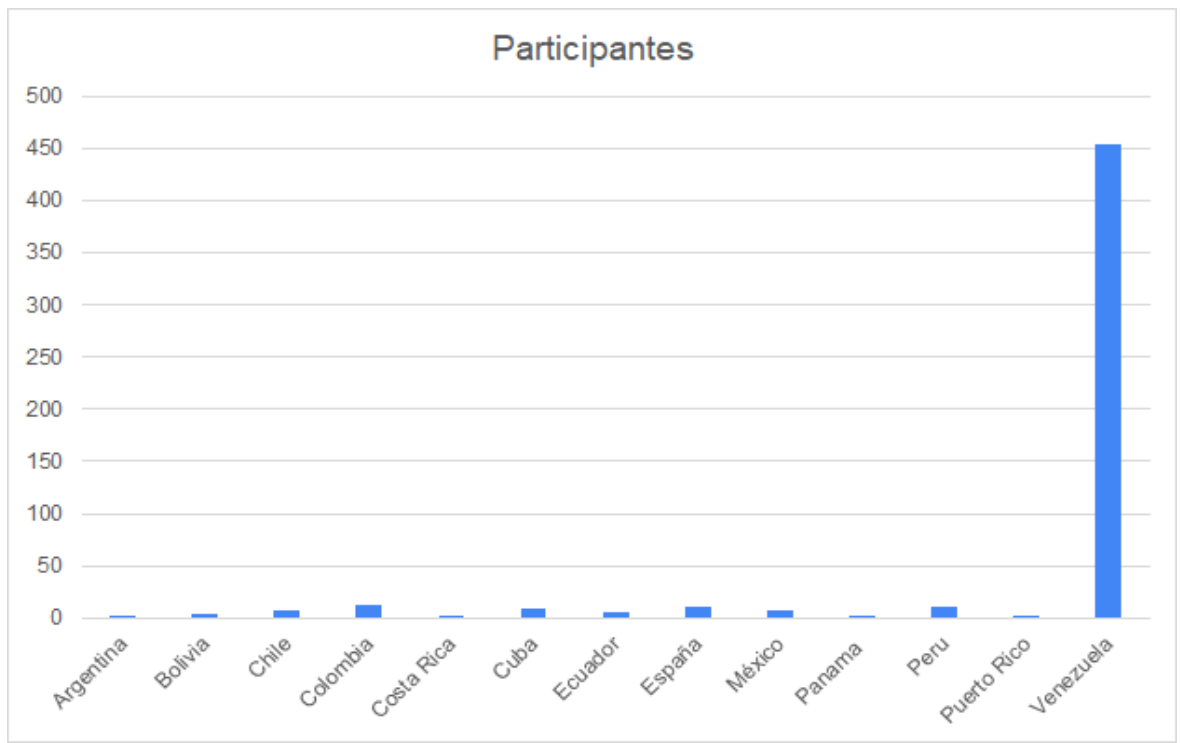

Gráfico 2. Fuente propia. 
Mediante el estudio de los participantes inscritos mediante el formulario online habilitado podemos observar que el desarrollo de la formación en los módulos mostró un elevado interés de los participantes en aquellos correspondientes al área didáctica y las herramientas tecnológicas tecnológicas más accesibles:

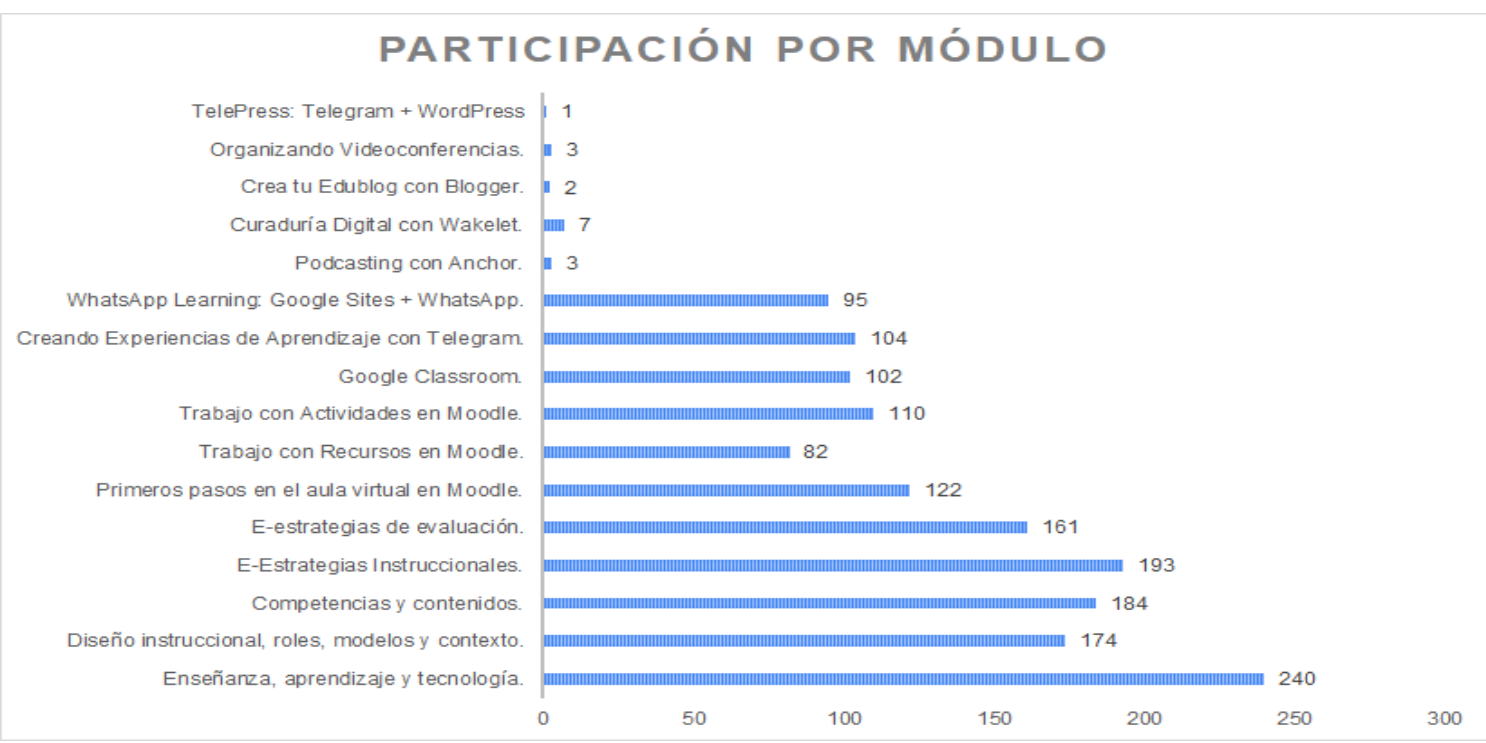

Gráfico 3. Fuente propia.

La muestra de estudio evidenció un alto porcentaje de participantes provenientes del sector universitario, aun cuando se contó además con la participación de profesionales independientes, empresas, gobierno, educación media en general y organizaciones no gubernamentales.

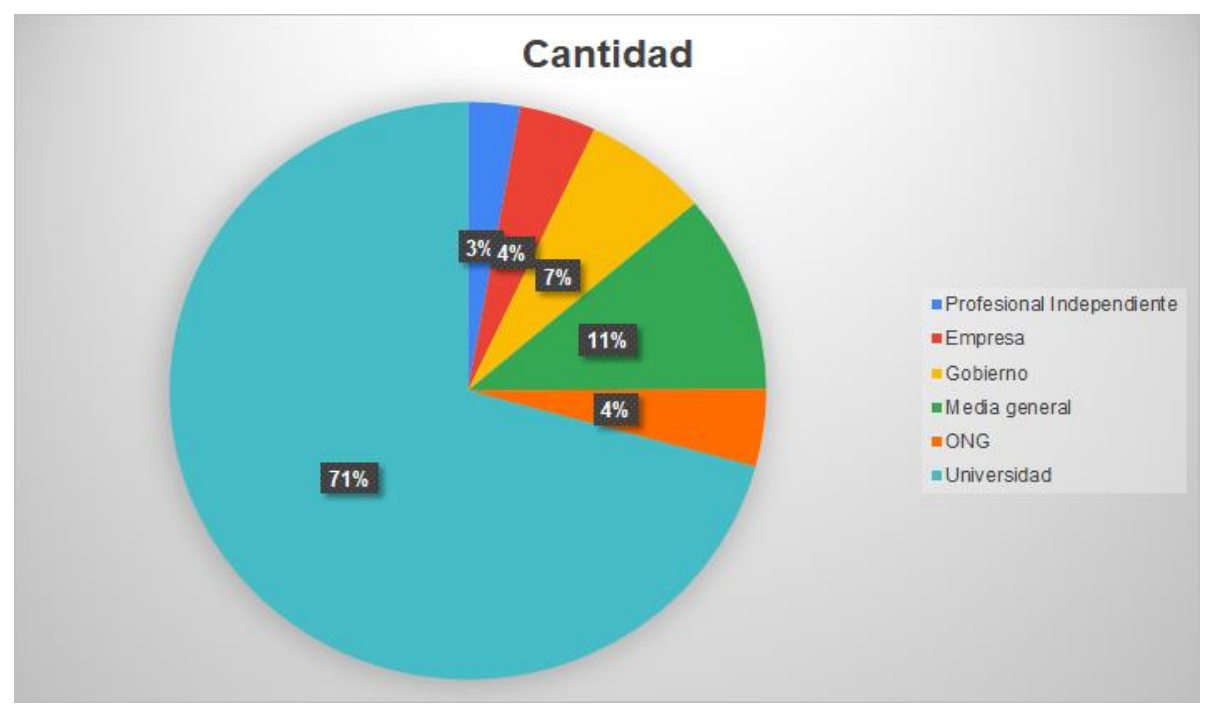

Gráfico 4. Fuente propia. 
Número de participantes que culminaron cada módulo

Enseñanza, aprendizaje y tecnología. Diseño instruccional, roles, modelos y...

Competencias y contenidos.

E-Estrategias Instruccionales.

E-estrategias de evaluación.

Primeros pasos en el aula virtual en...

Trabajo con Recursos en Moodle.

Trabajo con Actividades en Moodle.

Google Classroom.

Creando Experiencias de Aprendizaje c...

WhatsApp Learning: Google Sites +.

Podcasting con Anchor.

Curaduría Digital con Wakelet.

Crea tu Edublog con Blogger.

Organizando Videoconferencias.

TelePress: Telegram + WordPress
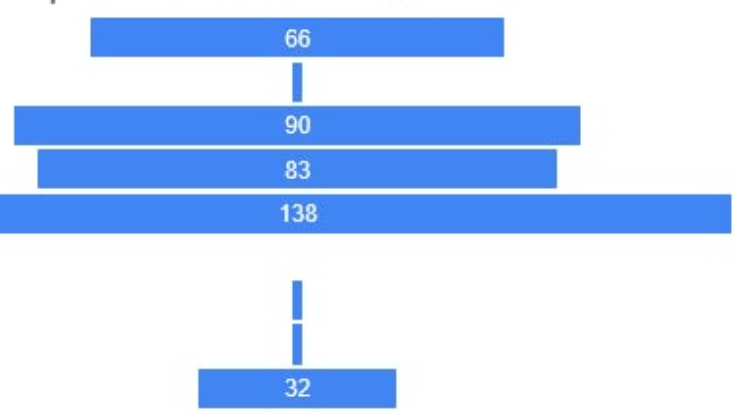

\section{Gráfico 5. Fuente propia.}

\section{Análisis de "Fortalezas, Debilidades, Oportunidades y Desafíos" (FODD)}

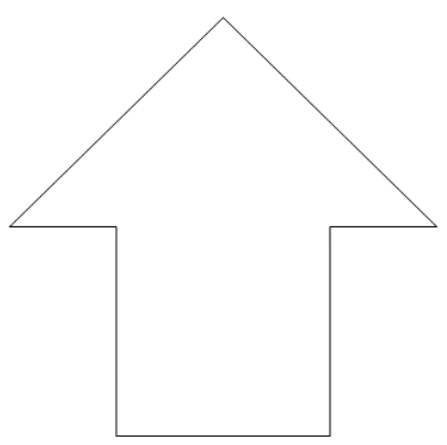

Fortalezas

- La propuesta recoge, estructura y organiza experiencias formativas previas evaluadas y

mejoradas.

-La organización y estructura en unidades didácticas constituye una forma efectiva para concretar,

presentar y gestionar el entorno virtual de enseñanza y aprendizaje.

-El equipo de trabajo está blindado y fortalecido en el dominio de competencias y saberes de

naturaleza didáctica y tecnológica.

- La propuesta ha brindado la posibilidad de construir redes y recibir avales de instituciones

académicas a nivel nacional: UCV- ULA

- La propuesta se vale del uso de una plataforma tecnológica estable de gestión de cursos en línea

- La modalidad asincrónica propuesta facilita la autogestión del aprendizaje.

- La secuencia en el diseño de la instrucción permite construir la ruta individual de aprendizaje

particular con base en sus necesidades de formación.

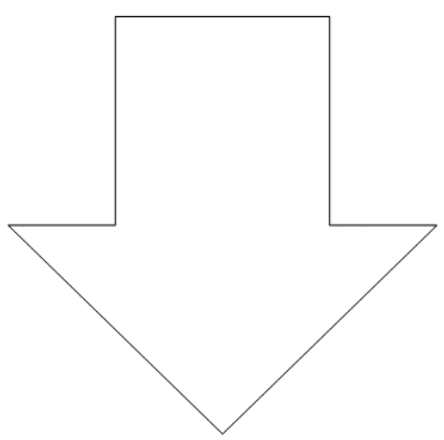

Debilidades

-El clúster no cuenta con suficientes monitores otutores.

-En el diseño de la experiencia formativa no se han propuesto espacios de comunicacióne interacción sincrónica para complementary/o profundizar en los contenidos.

- El seguimiento de instrucciones para el acceso, registro y gestión presenta fallas en algunos

-Hay una falla medular en la cultura del aprendizaje en cuanto a la autonomía del aprendizaje - No todos los participantes culminan y cierran las unidades didácticas cursadas. 


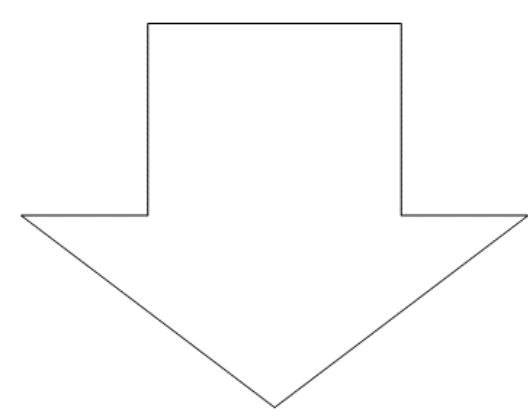

Oportunidades

- El confinamiento por el COVID que pone su foco en la educación a distancia mediada por las

tecnologías de la información y la comunicación.

- La necesidad de capacitación en el uso de las tecnologías de información y comunicación para el diseño y desarrollo de procesos e-learning, no solo en las instituciones educativas sino también en el ámbito de las empresas y gobierno.

- El amplio uso y adopción de los dispositivos móviles (teléfonos móviles y tabletas) con conectividad a internet que facilitan el impulso y consolidación del aprendizaje ubicuo.

\section{Desafíos}

- Conectividad a internet y disponibilidad de servicio eléctrico, en toda la región de impacto del CLEAP - 7

-Asunción de las pautas de autogestión del participante en su proceso de aprendizaje, en un diseño modular y flexible donde este debe tomar todas las decisiones para avanzar y culminar con éxito.

- Conformación y establecimiento de una comunidad de aprendizaje colaborativo en torno a cada uno de los módulos que conforman el clúster.

- La brecha digital que aún persiste en nuestros países y que se acentuó como consecuencia de la pandemia.

- Continuar promoviendo la apropiación conceptual y la praxis de la autogestión del aprendizaje y la creación de rutas de aprendizaje sobre la base de diagnóstico de necesidades de formación.

- Crear herramientas tecnológicas que permitan crear, registrar y da

seguimiento a la autogestión del aprendizaje y a sus respectivas rutas -Continuidad del proceso de formación post pandemia.

\section{Análisis de Factores Críticos del Éxito (FCE)}

Cuadro 1. Fuente propia

\begin{tabular}{|c|c|c|}
\hline $\begin{array}{l}\text { OBJETIVOS } \\
\text { CLEAP } 7\end{array}$ & $\begin{array}{l}\text { FACTORES } \\
\text { INTERNOS }\end{array}$ & $\begin{array}{l}\text { FACTORES } \\
\text { EXTERNOS }\end{array}$ \\
\hline $\begin{array}{l}\text { Ofrecer un programa } \\
\text { de acceso abierto y } \\
\text { masivo para la } \\
\text { formación } \\
\text { capacitación y } \\
\text { docente en el uso de } \\
\text { las TIC }\end{array}$ & $\begin{array}{l}\text { - Experiencia previa en } \\
\text { DDG de cursos de acceso } \\
\text { abierto y masivo. } \\
\text { - Plataforma de gestión } \\
\text { de cursos. }\end{array}$ & $\begin{array}{l}\text { - Necesidad de } \\
\text { formación y/o actualización } \\
\text { en el uso educativo de las } \\
\text { TIC'S para docentes en los } \\
\text { distintos niveles en L.A. } \\
\text { • Necesidades de } \\
\text { ofertas, formación y } \\
\text { capacitación gratuitas. }\end{array}$ \\
\hline $\begin{array}{l}\text { Reunir en el espacio } \\
\text { común virtual de } \\
\text { AULACIETE todos } \\
\text { los módulos } \\
\text { orientados a la } \\
\text { formación docente } \\
\text { en el uso de las TIC, }\end{array}$ & $\begin{array}{l}\text { - Plataforma propia de } \\
\text { gestión y administración } \\
\text { educativa } \\
\text { - Diseño y modelo } \\
\text { educativo propio y validado }\end{array}$ & $\begin{array}{l}\text { - Búsqueda } \\
\text { programas de } \\
\text { bajo e -learning } \\
\text { - Búsqueda } \\
\text { programas de formación } \\
\text { para el uso de herramientas } \\
\text { tecnológicas }\end{array}$ \\
\hline
\end{tabular}




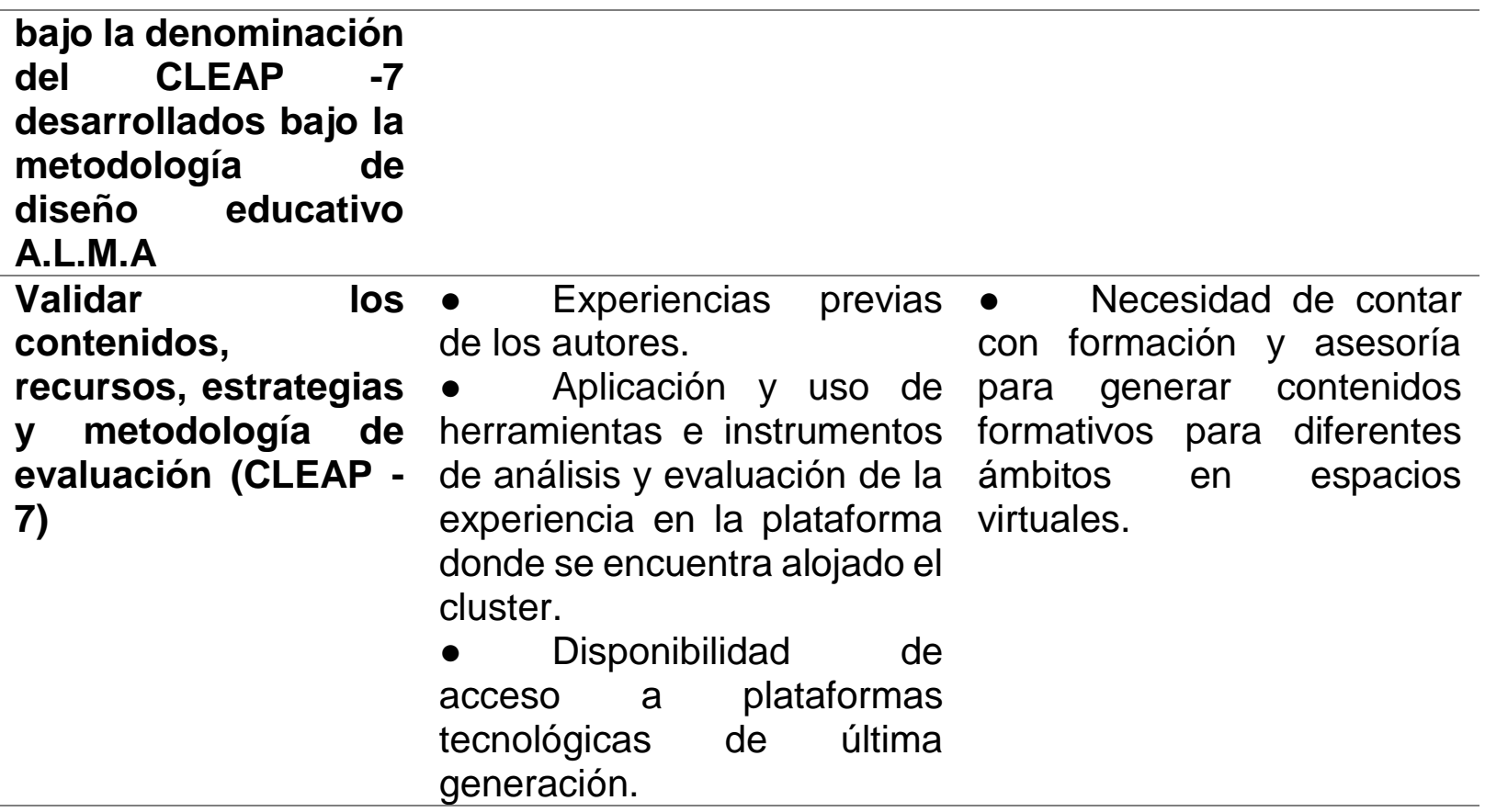

\section{Conclusiones}

Como síntesis de la implementación del CLEAP - 7 en la plataforma de AULACIETE entre septiembre 2020 y febrero 2021 se pudieron extraer las siguientes conclusiones:

Los elementos cardinales que conformaron la iniciativa para el proceso de desarrollo de la oferta educativa gratuita y de libre acceso CLEAP - 7 que se desarrolló entre septiembre 2020 y febrero 2021 están relacionados fundamentalmente con:

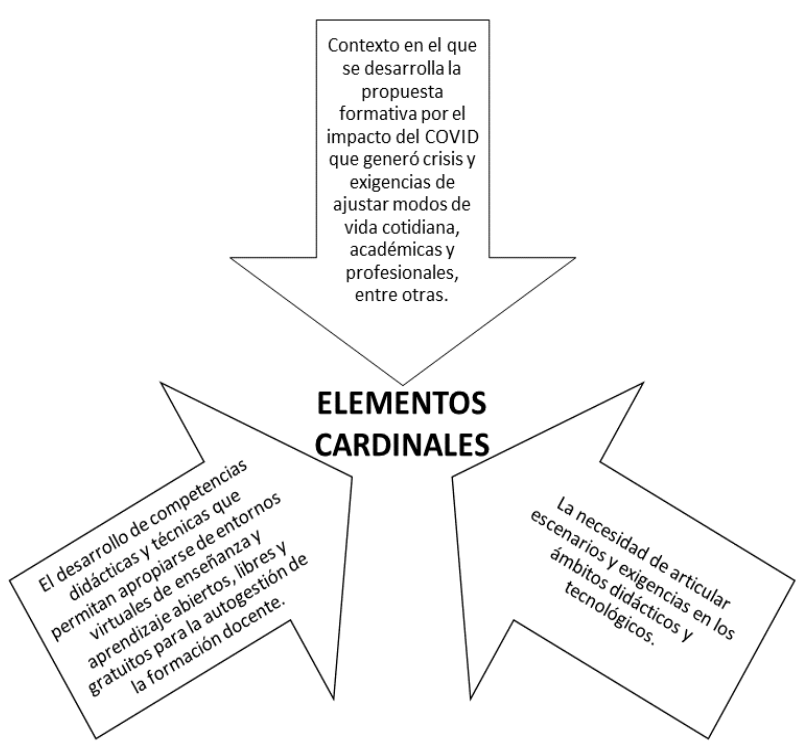

64 E-learning en tiempos de COVID. La experiencia del CLUSTER de Enseñanza -Aprendizaje AULACIETE 2020-2021. 
Nos permitimos resaltar de los resultados del análisis "Fortalezas, Debilidades, Oportunidades y Desafíos" (FDOD) lo siguiente:

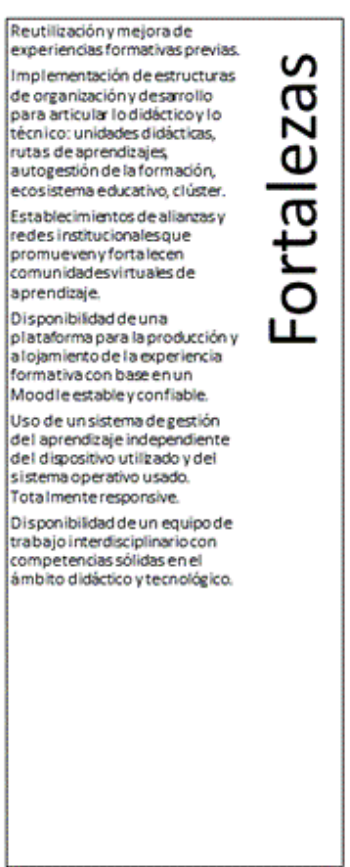

\section{ANÁLISIS FDOD}
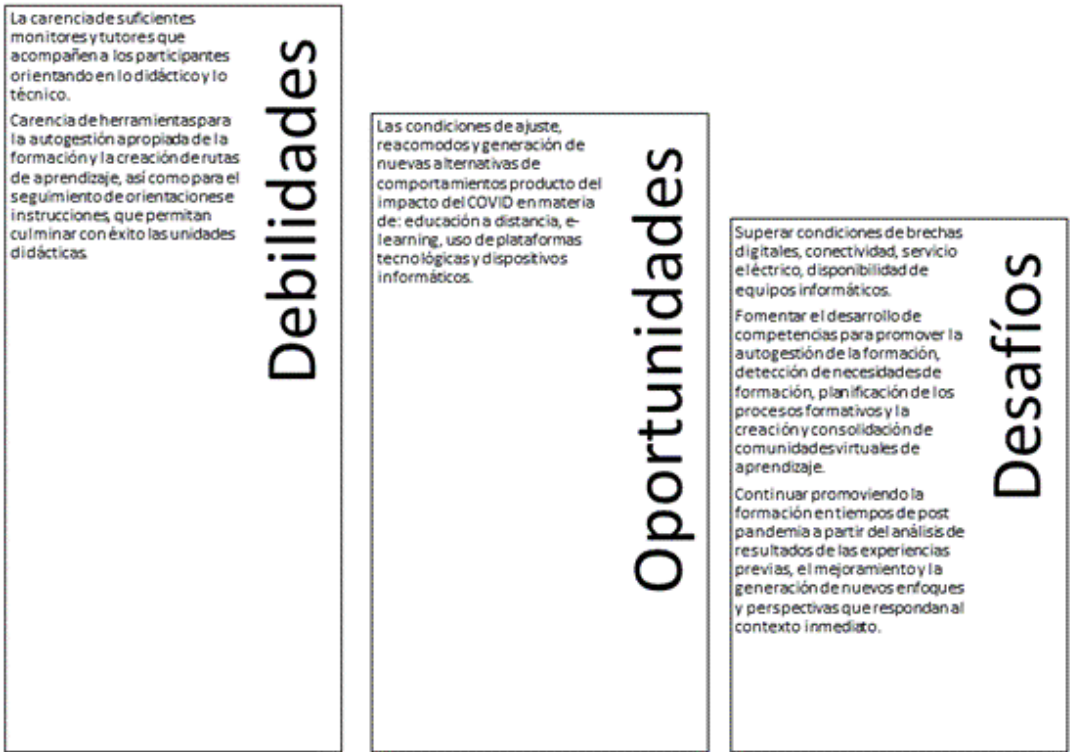

Es fundamental exponer que entre los "Factores Críticos de Éxito (FCE)" podemos concluir:

\section{FACTORES CRÍTICOS DE ÉXITO}

Articulación estratégica entre la existencia de experiencias formativas previas, de una plataforma de gestión de aprendizaje estable y de calidad y las necesidades de formación a distancia mediadas por las tecnologías de la información y la comunicación en tiempo de COVID.
Configurar en un solo espacio una experiencia formativa de un modelo didáctico y tecnológico propio con base en un ecosistema educativo, clúster, unidades didácticas y rutas de aprendizaje para la autogestión.
Búsqueda de opciones para mejorar y enriquecer procesos de monitoreo, seguimientoy tutoría virtual que fortalezcan los procesos de validación. 
Sugerimos y concluimos que:

- La modalidad de educación a distancia constituye un reto y una oportunidad que contribuye de forma efectiva a salvar las condiciones de vida impuestas por el COVID.

- Los docentes deben asumir la formación y la actualización como procesos que requieren la detección de necesidades de formación y la construcción de rutas de aprendizajes que permitan la autogestión de su formación.

- La autogestión de la formación y la creación de sus rutas de aprendizaje contribuyen a satisfacer de forma efectiva necesidades formativas reales.

- La autogestión de la formación y la creación de sus rutas de aprendizaje contribuyen a orientar la toma de decisiones ante la variada y masiva oferta formativa en la modalidad a distancia.

- El éxito de la formación a distancia en su forma de e- learning requiere de condiciones mínimas de dotación de equipos informáticos, programas, aplicaciones especializadas, servicios de conectividad y eléctricos estables.

- Se requiere promover espacios y experiencias para la apropiación y desarrollo de competencias didácticas y técnicas que permitan la autogestión del aprendizaje en entornos virtuales de enseñanza y aprendizaje modulares, abiertos y flexibles.

- Se confirma la necesidad e importancia del diseño instruccional como proceso de planificación didáctica que contribuye a garantizar la calidad de la enseñanza y el aprendizaje en la articulación de exigencias académicas y técnicas.

- La unidad didáctica constituye una forma de organización y estructura de planificación que permite la articulación y dosificación de procesos y componentes didácticos y técnicos

\section{Referencias}

Alvarado, A., \& Marquina, R. (2020) "Cluster de enseñanza Aprendizaje" https://aulaciete.net/cleap7/

Alqahtani, Y., \& Rajkhan, A. (2020) "E-Learning Critical Success Factors during the COVID-19 Pandemic: A Comprehensive Analysis of E-Learning Managerial Perspectives" Educ. Sci., 10(9), 216, https://doi.org/10.3390/educsci10090216 https://www.mdpi.com/2227-7102/10/9/216/htm

Arias Ortiz, E., \& Viteri, A. (2019) ¿Cuentan las escuelas con la tecnología necesaria para la transformación digital? Inter-American Development Bank. https://doi.org/10.18235/0001629

Belazi, H. M., Rubin, E. J., \& Toribio, A. J. (2013). Code switching and X-bar theory: The functional head constraint. Linguistic inquiry, 221-237. CEPAL - UNESCO (2020) "La educación en tiempos de la pandemia de COVID -19" Naciones Unidas. https://repositorio.cepal.org/bitstream/handle/11362/45904/1/S2000510_es.pdf

Cabero, J., \& Almudena, G (2019) "Las tecnologías de la información y la comunicación y la formación inicial de los docentes. Modelos y Competencias digitales. Revista Profesorado, 23(3) (JULIO-SEPTIEMBRE, 2019) ISSN 1138-414X, DOI:

E-learning en tiempos de COVID. La experiencia del CLUSTER de Enseñanza -Aprendizaje AULACIETE 2020-2021. 
10.30827/profesorado.v23i3.9421

https://recyt.fecyt.es/index.php/profesorado/article/view/74491

Castañeda, L., Fracesc, E. \& Adell, J (2018) ¿Por qué es necesario repensar la competencia docente para el mundo digital? RED. Revista de Educación a Distancia. Núm. 56, Artíc. 6, 31-01-2018. DOI: http://dx.doi.org/10.6018/red/56/6; https://www.um.es/ead/red/56/castaneda_et_al.pdf

Corrales, A. (2010). La programación a medio plazo dentro del tercer nivel de concreción: las unidades didácticas. Emás F, Revista Digital de Educación Física, Año 1, Núm. 2 (enero-febrero 2010), https://dialnet.unirioja.es/servlet/articulo?codigo=3175435

García Aretio, L. (2021). COVID-19 y educación a distancia digital: preconfinamiento, confinamiento y posconfinamiento. RIED. Revista Iberoamericana de Educación a Distancia, 24(1), pp. 09-32. doi: http://dx.doi.org/10.5944/ried.24.1.28080

García Aretio, L. (2020). Una Unidad Didáctica, un Tema, una Lección en formato digital. Contextos universitarios mediados. (ISSN: 2340-552X). Recuperado de https://aretio.hypotheses.org/1933

Hodges, C., Moore, S., Lockee, B., Trust, T., y Bond, A. (2020). La diferencia entre la enseñanza remota de emergencia y el aprendizaje en línea. https://er.educause.edu/articles/2020/3/the-difference-between-emergencyremote-teaching-and-online-learning

Marquina, R. Alvarado, A. (2019) "Accuracy Learning by Modular Acknowledgment A.L.M.A. Modelo de I+D+D de programas de formación y capacitación para el ámbito académico y empresarial", https://aulaciete.net/alma

Ponce, M. (2016). La autogestión para el aprendizaje en estudiantes de ambientes mediados por tecnología. Revista Diálogos sobre educación. Temas actuales en investigación educativa, 7(12), 2016. Universidad de Guadalajara, México, http://www.redalyc.org/articulo.oa?id=553458153013

Reimers, F. M., \& Schleicher, A. (2020). "A framework to guide an education response to the COVID-19 Pandemic of 2020" OECD - PISA https://oecd.dambroadcast.com/pm_7379_126_126988-t63lxosohs.pdf

Rojas-Bahamón, M. J., Aguilar-Cruz, P.J., \& Arbeláez-Campillo, D.F. (2020). Curricular integration as a strategy to strengthen the educational process in public institutions in COVID-19 times. Revista Inclusiones, 7(num Especial), 233-241. http://www.revistainclusiones.org/index.php/inclu/article/view/1503

Singh, V.; Thurman, A. How many ways can we define online learning? A systematic literature review of definitions of online learning (1988-2018). Am. J. Distance Educ., 33, 289-306.Open Access Artícle. MDPI. Education Science. https://www.mdpi.com/2227-7102/10/9/232/htm

Sivagni, D. (2020) "Online learning. A Panacea in the time of COVID 19 Crisis" https://journals.sagepub.com/doi/full/10.1177/0047239520934018

https://doi.org/10.1177/0047239520934018

Sistema de Información de Tendencias Educativas en América Latina "SITEAL" (2020) Informe octubre https://siteal.iiep.unesco.org/respuestas_educativas_covid_19

2020. 
UNESCO (2020) "La educación en América Latina y el Caribe". https://es.unesco.org/fieldoffice/santiago/covid-19-education-alc/respuestas

UNESCO (2020) "Informe de sistematización de los sistemas educativos de América Latína a la crisis de la COVID 19" https://es.unesco.org/fieldoffice/santiago/covid19-education-alc/respuestas)

UNESCO (2019)"Marco de competencias de los docentes en materia de TIC, París" https://unesdoc.unesco.org/ark:/48223/pf0000371024.

UNICEF (2020) "Plataformas y recursos educativos en línea Listado de las plataformas y recursos de aprendizaje disponibles en América Latina y el Caribe" https://uni.cf/3bf3g6G 